\title{
UV laser induced changes to morphological, optical and electrical properties of conductive nanocrystalline diamond films
}

\author{
Maxim S. Komlenok ${ }^{1,2}$, Anna M. Zaniewski ${ }^{3}$, Evgeny V. Zavedeev ${ }^{1,2}$, Vitaly I. Konov ${ }^{1,2}$ \\ Franz A.M. Koeck ${ }^{3}$, Robert J. Nemanich ${ }^{3}$ \\ ${ }^{1}$ Natural Sciences Center, General Physics Institute, Vavilova 38, Moscow, 119991, Russia \\ ${ }^{2}$ National Research Nuclear University MEPhI (Moscow Engineering Physics Institute), \\ Kashirskoye shosse 31, Moscow 115409, Russia \\ ${ }^{3}$ Department of Physics, Arizona State University, Tempe AZ, 85287-1504 USA
}

\begin{abstract}
Keywords
Conductive nanocrystalline diamond; nanoablation; laser; AFM
\end{abstract}

\begin{abstract}
The possibility of laser induced variation of optical and electrical properties of conductive nanocrystalline diamond (CNCD) films has been demonstrated. The films were produced by microwave plasma chemical vapor deposition (MPCVD) from $\mathrm{CH}_{4}: \mathrm{H}_{2}: \mathrm{N}_{2}$ gas mixtures. The films were irradiated in air with $20 \mathrm{~ns}$ pulses of an $\operatorname{ArF}$ excimer laser $(\lambda=193 \mathrm{~nm})$. It was found that low laser pulse intensity $\left(\sim 0.05 \mathrm{~J} / \mathrm{cm}^{2}\right)$, well below film surface graphitization $\left(\sim 0.3 \mathrm{~J} / \mathrm{cm}^{2}\right)$ and nanoablation $\left(\sim 0.08 \mathrm{~J} / \mathrm{cm}^{2}\right)$ thresholds, induces changes of the film properties. The effect requires multiple pulsed irradiation and results in a decrease of the film electrical conductivity, which is accompanied by optical bleaching of the diamond film absorption.
\end{abstract}

\section{Introduction.}

Conductive nanocrystalline diamond (CNCD) films have great potential as a new material for electronic devices, e.g. as field electron emitters for displays and as thermionic emitters for energy conversion applications [1,2]. Such films can be produced by microwave plasma chemical vapor deposition (MPCVD) synthesis with the addition of nitrogen to the traditional $\mathrm{CH}_{4}: \mathrm{H}_{2}$ based gas. The films show electrical conductivity with n-type character [3]. The nitrogen incorporation has been shown to be largely in the grain boundaries [4], and 


\section{Experiment}

CNCD films were deposited on mirror polished $25 \mathrm{~mm}$ diameter conductive silicon (001) substrates (P-doped, n-type, $0.01 \Omega$-cm, from Virginia Semiconductor) using $\mathrm{N}_{2}: \mathrm{H}_{2}: \mathrm{CH}_{4}$ gas mixtures by MPCVD [1]. The films were prepared with a $\mathrm{N}_{2}$ gas phase concentration greater than $20 \%$, which corresponds to a $[\mathrm{N}]$ concentration of $\sim 2 \times 10^{20} \mathrm{~cm}^{-3}$ and a resistivity of $\sim 0.1$ $\Omega-\mathrm{cm}[8,9]$. The initial film thickness was $\sim 150 \mathrm{~nm}$. For laser processing, the samples were mounted on a holder attached to a translation stage. An ArF excimer laser (wavelength of 193 $\mathrm{nm}$, pulse duration of $20 \mathrm{~ns}$ and a repetition rate of $50 \mathrm{~Hz}$ ) was used for sample irradiation at ambient atmospheric conditions. The beam was focused on a rectangular aperture to establish the exposed area of the sample surface. The laser pulse fluence, E, was varied from 0.05 to $5 \mathrm{~J} / \mathrm{cm}^{2}$. The film properties were analyzed before and after laser treatment. Surface morphology was investigated by standard (Carl Zeiss, Axiotech 25 HD) and white light interferometric (Zygo, New View 5000) optical microscopes as well as scanning probe microscopes (NT-MDT, NTEGRA Solaris, Asylum MFP3D). We utilized a micro-Raman spectrometer (Jobin Yvon, LabRAM HR800) with an excitation wavelength of $488 \mathrm{~nm}$ to determine the type of carbon 
bonding present. The spatial uniformity of the film conductivity was measured with an Asylum atomic force microscope operated with a conductive diamond nanoprobe.

\section{Results and discussion}

We have found that at low laser fluence $\left(\mathrm{E}=\mathrm{E}_{\mathrm{o}} \approx 0.05 \mathrm{~J} / \mathrm{cm}^{2}\right)$, which is below the nanoablation threshold for CNCD films, pulsed laser exposure can induce changes to the optical and electrical properties of the diamond film without evident crater formation. To observe such changes of material properties, multiple (pulse number $\mathrm{N}>10^{4}$ ) laser pulses are needed. Using an optical microscope, one can visually observe the difference in colour of the irradiated regions of the sample (bright image on the left side in Fig. 1a) and the initial unprocessed regions of the film (right side in Fig. 1a). The corresponding atomic force microscope images of the film surface morphology prior to and after multiple pulsed laser irradiation exposures $\left(\mathrm{E}_{\mathrm{o}} \approx 0.05\right.$ $\mathrm{J} / \mathrm{cm}^{2}$ and $\mathrm{N}=3 \cdot 10^{4}$ pulses) are presented in Fig. 1b. It is evident that the film surface shows a similar morphology before and after the laser irradiation. The size and distribution of nanoprotrusions and nanopores remains the same. Moreover, the average surface roughness does not change and equals to $\mathrm{R}_{\mathrm{a}} \approx 30 \mathrm{~nm}$. Therefore, we can conclude that laser induced variation of the CNCD film surface morphology, which can influence light scattering, is not responsible for the observed optical effect.

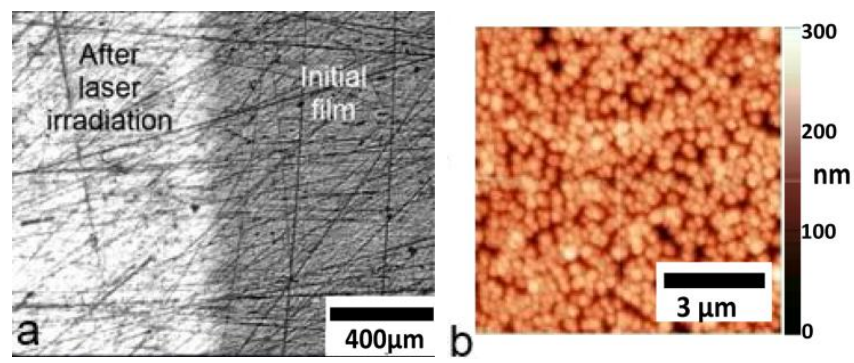

Fig. 1 Images of CNCD film from (a) an optical microscope and (b) an AFM. The left part of both images corresponds to the film after laser irradiation with a fluence of $0.05 \mathrm{~J} / \mathrm{cm}^{2}$ and $N=$ $3 \cdot 10^{4}$ pulses. 


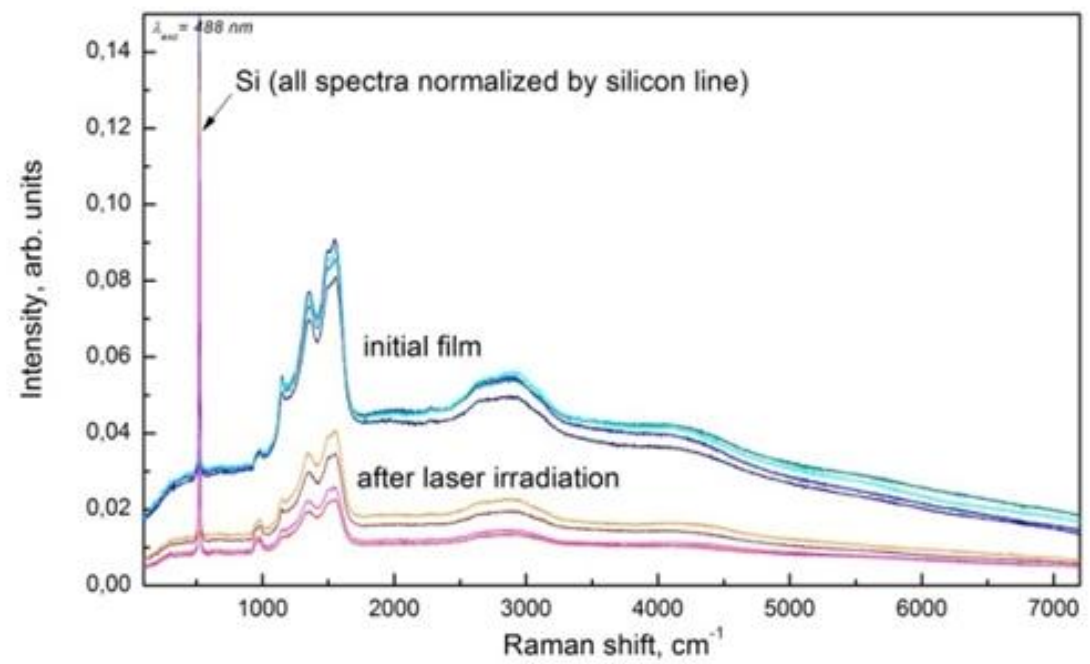

Fig. 2. Raman spectra of diamond film before and after laser irradiation. All spectra normalized to the $520 \mathrm{~cm}^{-1}$ silicon line.

Raman spectra before and after laser treatment at $\mathrm{E}=\mathrm{E}_{0}$ are presented in Fig. 2. The different scans represent different regions on the sample. The silicon line from the substrate is clearly visible at $520 \mathrm{~cm}^{-1}$, and all of the spectra have been normalized to the intensity of this line. Vibrational modes of $\mathrm{sp}^{2}$ bonded carbon $\mathrm{D}\left(1360 \mathrm{~cm}^{-1}\right)$ and $\mathrm{G}\left(1590 \mathrm{~cm}^{-1}\right)$ and second order Raman features around 2700-2900 $\mathrm{cm}^{-1}$ are evident [16]. The broad background has been attributed to defect related luminescence [17]. The relative intensity and the shape of the carbon lines remains essentially unchanged, but the relative intensity decreases by about $50 \%$ after laser irradiation $\left(E=E_{0}, N=3 \cdot 10^{4}\right)$. The absolute intensity of the $520 \mathrm{~cm}^{-1} \mathrm{Si}$ substrate signal increases by about $30 \%$ while the absolute intensity of the $\mathrm{sp}^{2}$ related features decreases by about $30 \%$. Consequently, the two effects contribute approximately equally to the observed relative change in the ratio of the silicon substrate line intensity, $I_{s i}$, to that of the $D$ and G peaks, $I_{D(G)}$. We note that both the bleaching and the strong decrease of the $\mathrm{sp}^{2}$ signal after laser treatment, have occurred while the thickness of the film is essentially unchanged. Evidently, the decrease in the optically absorbing $\mathrm{sp}^{2}$ bonded content can account for the film bleaching effect.

The differences in optical properties of the initial and laser treated films may be explained by the laser induced variation of carbon phases and their ratio [18]. There are two effects that could be considered: 1) surface oxidation, and 2) redistribution of the hydrogen in the film. First we estimate the surface temperature due to the laser pulse process. For a diamond film in ambient air, surface graphitization is known to occur at a temperature $\mathrm{T}_{\mathrm{g}} \approx 800^{\circ} \mathrm{C}$. The fluence 
range employed in our experiments was varied from 0.05 through $5 \mathrm{~J} / \mathrm{cm}^{2}$, and the ablation rate was observed to decrease by more than 2 orders of magnitude at a fluence of $\mathrm{E} \approx \mathrm{E}_{\mathrm{g}} \approx 0.3 \mathrm{~J} / \mathrm{cm}^{2}$, which is typical for this change of the mechanism of the diamond film ablation [14]. High-rate ablation is resulted from evaporation of graphitized surface layer, and low-rate etching (nanoablation) is characterized by laser-induced oxidation of initial material. So we can use this threshold value to estimate the surface temperature at $\mathrm{E}=\mathrm{E}_{0}$. We assuming a linear relationship between temperature and laser power which assumes the optical properties are constant in this range. Then the estimated temperature at $\mathrm{E}=\mathrm{E}_{0}$ is found to be $\mathrm{T}_{0} \approx \mathrm{T}_{\mathrm{g}}\left(\mathrm{E}_{0} / \mathrm{E}_{\mathrm{g}}\right) \approx 130^{\circ} \mathrm{C}$. We note that this is a conservative but realistic approach since the optical absorption decreases slightly during the measurements. At this low temperature one would not expect significant film oxidation that could lead to the removal of a significant quantity of the film. Prior research has shown that low intensity pulsed illumination with UV radiation can induce oxidation of carbon nanodots [18] and of highly oriented pyrolitic graphite [19]. Consequently, the UV illumination can induce oxidation of small clusters or surface atoms of $\mathrm{sp}^{2}$ bonded carbon. The optical properties of the structures can be changed and/or the reacted atoms can be chemically removed as $\mathrm{CO}$ or $\mathrm{CO}_{2}$ from the surface and intergranular space. As a result fewer $\mathrm{sp}^{2}$ bonded carbon atoms remain on the film surface. Considering the film porosity and the high optical absorption of the $\mathrm{sp}^{2}$ phase, the low intensity UV illumination could account for the diamond film bleaching by oxidization of $\mathrm{sp}^{2}$ clusters not only at the thin surface layer, but throughout the film thickness.

In an oxidizing atmosphere (air) the graphitic phase domains are expected to be thermally etched at a faster rate than the diamond domains. Taking into account: (i) the exponential dependence of the chemical etching rate vs. temperature; (ii) the short oxidation time as a result of ns laser pulses; and (iii) the diffusion limitation for oxygen molecules to reach the film surface during the short reaction time, it becomes quite reasonable to consider that graphitic phase etching under the considered conditions should be very small and a larger number of laser pulses would be needed for a pronounced optical effect, as observed.

Another possible explanation for the reduction of the film optical absorption is a change of the hydrogen distribution in the film. The nanocrystalline diamond films are known to have $\mathrm{H}-$ concentrations of up to $10 \%$ [20], part of which is bonded in polymeric carbon configurations at grain boundaries [21]. We speculate that the pulsed laser process may induce a redistribution of the hydrogen in the film [22], thus causing a change of carbon hybridization from $\mathrm{sp}^{2}$ to $\mathrm{sp}^{3}$ 
configurations.

Since graphitic material has a large visible light absorption coefficient of $10^{5} \mathrm{~cm}^{-1}$ either removal of $\mathrm{sp}^{2}$ bonded regions or conversion to $\mathrm{sp}^{3}$ could provide a large enough effect to make the film more transparent in the visible spectral range. The observation of optical bleaching of the CNCD film is in agreement with prior results on irradiation of diamond films with $<10 \mathrm{~nm}$ grains and notable $\mathrm{sp}^{2}$ and $\mathrm{sp}^{3}$ components (UNCD films). In this study saturation of the etching rate was observed at increased numbers of pulses [23].

Another argument for laser induced carbon structure modification of the films at $\mathrm{E}=\mathrm{E}_{0}$ is the changing of the film electrical conductivity - this effect was observed in our experiments by means of a conductive diamond AFM cantilever. We chose AFM to measure conductivity to determine local, spatially resolved, changes in conductivity. Again we found a difference between the initial and laser modified film areas, as can be observed in Fig. 3a (the boundary between the two areas is in the middle). The amplitude of electric current averaged over a 5 micron region is displayed in Fig. 3c. Outside the laser modified spot the current is 360 pA, while inside the spot it is $25 \mathrm{pA}$ at $10 \mathrm{~V}$. Consequently, more than an order of magnitude drop of film conductivity was observed after the pulsed laser treatment. Consistent with the optical changes, this modification of the film electrical resistance likely represents a decrease in the band conductivity through $\pi$-bonded states $[7,8]$. This change could be realized with partial removal of the conductive $\mathrm{sp}^{2}, \pi$-bonded regions or conversion of $\mathrm{sp}^{2}$ into $\mathrm{sp}^{3}$ bonded carbon regions. Since the energy of the laser $(6.4 \mathrm{eV})$ is above the bandgap of diamond $(5.5 \mathrm{eV})$ and the film is the mixture of $\mathrm{sp}^{2}$ and $\mathrm{sp}^{3}$ phases, transmission to the substrate is minimal, and we can assume that the conductivity changes are a reflection of changes to the film, not the highly conductive substrate or interface. 
Fig. 3. Conductivity (a) and morphology (b) measurements of diamond film by use of AFM. The left side of the scans correspond to the initial film and right parts to the spot after laser irradiation. (c) Current and height vs distance across the boundary between pristine and irradiated parts of the CNCD film. (d) a schematic of conductive AFM. To make a complete circuit, the CNCD film and native silicon oxide were removed from the silicon substrate with mechanical etching just outside the region of interest, and silver paint was applied to provide a high quality contact.

At slightly higher laser fluence $\left(\mathrm{E}=\mathrm{E}_{\mathrm{a}}=0.08 \mathrm{~J} / \mathrm{cm}^{2}\right)$ crater formation is observed after film irradiation in air. Fig. 4 represents a profile of such a crater in the CNCD film after 1000 laser pulses with $\mathrm{E}=\mathrm{E}_{\mathrm{a}}$. The depth of the crater is only about $30 \mathrm{~nm}$, which is less than the 150 $\mathrm{nm}$ film thickness. This is a new example of pulsed laser induced surface oxidation (nanoablation), which was earlier observed for non-conductive diamond samples [13, 14]. The average etching rate is only $0.03 \mathrm{~nm} /$ pulse, which allows application of a nanoablation process 
for ultra-precise film nanostructuring. For example, about 100 laser pulses is needed to form a $\sim 3$ $\mathrm{nm}$ deep crater. Note that with modern laser systems, e.g. disk lasers with $\mathrm{MHz}$ pulse repetition rates, nanostructure fabrication using a fast scanning laser beam is a scalable process.

For laser fluence $\mathrm{E}>\mathrm{E}_{\mathrm{g}}$ vaporization of a graphitized surface $\left(\mathrm{T} \approx 4000^{\circ} \mathrm{C}\right)$ can be realized. This is a typical physical ablation regime with crater formation rates above 10 $\mathrm{nm} / \mathrm{pulse}$, which can be realized for single or several laser pulse processes.

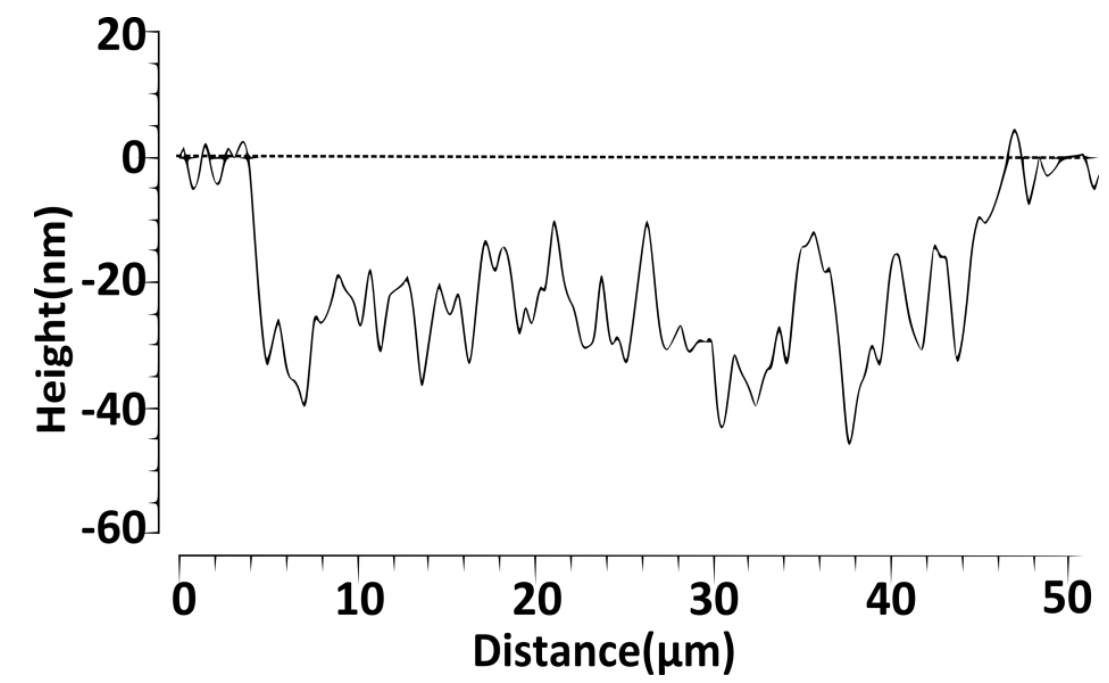

Fig. 4. Profile of a crater after 1000 laser pulses with $E=E_{a}$ obtained. The profile was obtained with an WLI microscope.

\section{Conclusions}

It is shown that depending on ArF laser pulse fluence and laser pulse number, different effects can be induced on the surface of nitrogen-doped nanocrystalline films:

1) For high pulse number $N \geq 10^{3}-10^{4}$ :

- film optical bleaching and a conductivity reduction at $\mathrm{E}=0.05 \mathrm{~J} / \mathrm{cm}^{2}$;

- film chemical etching (nanoablation) with rates as low as $10^{-2} \mathrm{~nm} /$ pulse at $\mathrm{E}=0.08$ $\mathrm{J} / \mathrm{cm}^{2}$.

2) For single and several pulse processes at $E$ exceeding $0.3 \mathrm{~J} / \mathrm{cm}^{2}$, surface graphitization and vaporization (physical ablation) takes place with average material removal rates $\geq 10$ $\mathrm{nm} / \mathrm{pulse}$.

Acknowledgements. The authors acknowledge A.A. Khomich from GPI (Russia) for Raman measurements. The travel expenses needed to fulfill bilateral scientific collaboration was 
Research activities in Russia were supported by the Russian Science Foundation under Grant 1422-00243, in the USA by the National Science Foundation under Grant DMR-1206935.

\section{References:}

1. Koeck, F.A.M. and R.J. Nemanich, Substrate-diamond interface considerations for enhanced thermionic electron emission from nitrogen doped diamond films. Journal of Applied Physics, 2012. 112(11).

2. Koeck, F.A.M., Nemanich, R.J., Balasubramaniam, Y., Haenen, K., Sharp, J., Enhanced thermionic energy conversion and thermionic emission from doped diamond films through methane exposure. Diamond and Related Materials, 2011. 20(8): p. 1229-1233.

3. Bhattacharyya, S., Auciello, O., Birrell, J., Carlisle, J. A., Curtiss, L. A., Goyette, A. N., Gruen, D. M., Krauss, A. R., Schlueter, J., Sumant, A., Zapol, P., Synthesis and characterization of highly-conducting nitrogen-doped ultrananocrystalline diamond films. Applied Physics Letters, 2001. 79(10): p. 1441-1443.

4. Liu, Y.L., Sun, K.W., Lin, Y.J.,Fong, S.-C., Lin, I.N., Tai, N.H., Microwave plasmaassisted photoluminescence enhancement in nitrogen-doped ultrananocrystalline diamond film. AIP Advances, 2012. 2(2): p. 022145.

5. Zapol, P., Sternberg, M., Curtiss, L.A., Frauenheim, T., Gruen, D.M., Tight-binding molecular-dynamics simulation of impurities in ultrananocrystalline diamond grain boundaries. Physical Review B, 2001. 65(4): p. 045403.

6. Vlasov, I.I., Kanzyuba, M.V., Shiryaev, A.A., Volkov, V.V., Ral'chenko, V.G., Konov, V.I., Percolation model of an insulator-conductor transition in ultrananocrystalline diamond films. JETP Letters, 2012. 95(7): p. 391-395.

7. Bhattacharyya, S., "Mechanism of high n-type conduction in nitrogen-doped nanocrystalline diamond," Phys. Rev. B 70, (2004) 125412.

8. Arenal, R., Bruno, P., Miller, D.J., Bleuel, M., Lal J., and Gruen, D.M., "Diamond nanowires and the insulator-metal transition in ultrananocrystalline diamond films," Phys. Rev. B 75, (2007) 195431.

9. Beloborodov, I. S., Zapol, P., Gruen, D. M., Curtiss, L. A., "Transport properties of ntype ultrananocrystalline diamond films,” Phys. Rev. B 74, (2006) 235434.

10. Sugioka, K., M. Meunier, and A. Piqué, Laser precision microfabrication. Vol. 135. 2010: Springer.

11. Kononenko, T.V., Komlenok, M.S., Pashinin, V.P., Pimenov, S.M., Konov, V.I., Neff, M., Romano, V., Luthy, W., Femtosecond laser microstructuring in the bulk of diamond. Diamond and Related Materials, 2009. 18(2-3): p. 196-199.

12. Okuchi, T., Ohfuji, H., Odake, S., Kagi, H., Nagatomo, S., Sugata, M., Sumiya, H., Micromachining and surface processing of the super-hard nano-polycrystalline diamond by three types of pulsed lasers. Applied Physics A, 2009. 96(4): p. 833-842.

13. Kononenko, V.V., Komlenok, M.S., Pimenov, S.M., Konov, V.I., Photoinduced laser etching of a diamond surface. Quantum Electronics, 2007. 37(11): p. 1043-1046.

14. Komlenok, M.S., Kononenko, V.V., Ralchenko, V.G., Pimenov, S.M., Konov, V.I., Laser Induced Nanoablation of Diamond Materials. Physics Procedia, 2011. 12(Part 2): p. 37-45.

15. Konov, V.I., Laser in micro and nanoprocessing of diamond materials. Laser \& 
Photonics Reviews, 2012. 6(6): p. 739-766.

16. Nemanich, R.J. and S.A. Solin, First- and second-order Raman scattering from finite-size crystals of graphite. Physical Review B, 1979. 20(2): p. 392-401.

17. Bergman, L., McClure, M.T.,Glass, J.T., Nemanich, R.J., The origin of the broadband luminescence and the effect of nitrogen doping on the optical properties of diamond films. Journal of Applied Physics, 1994. 76(5): p. 3020-3027.

18. Tan, D., Zhou, S., Shimotsuma, Y., Miura, K., Qiu, J., Effect of UV irradiation on photoluminescence of carbon dots. Optical Materials Express, 2014. 4(2): p. 213-219.

19. V.D. Frolov, P.A. Pivovarov, E.V. Zavedeev, M.S. Komlenok, V.V. Kononenko, V.I. Konov, Laser nanoablation of graphite, Applied Physics A-MATERIALS SCIENCE \& PROCESSING, 2014. 114 (1) p. 51-55.

20. Ballutaud, D., Jomard, F., Kociniewski, T., Rzepka, E., Girard, H., Saada, S., Sp3/sp2 character of the carbon and hydrogen configuration in micro- and nanocrystalline diamond. Diamond and Related Materials, 2008. 17(4-5): p. 451-456.

21. Ferrari, A.C. and J. Robertson, Origin of the 1150- $\mathrm{cm}^{-1}$ Raman mode in nanocrystalline diamond. Physical Review B, 2001. 63(12): p. 121405.

22. Radosinski, L, Kuchta, B., "Hydrogen chemisorption on carbin structures with mixed sp2-sp3 hybridization: empirical potential studies," Adsorption 20 (2014) 875.

23. Komlenok, M.S., Kononenko, V.V., Vlasov, I.I., Ralchenko, V.G., Arutyunyan, N.R., Obraztsova, E.D., Konov, V.I., Laser "Nano" ablation of Ultrananocrystalline Diamond Films. Journal of Nanoelectronics and Optoelectronics, 2009. 4(2): p. 286-289. 


\section{Increased \\ Initial film \\ After laser treatment} transparency

UV laser

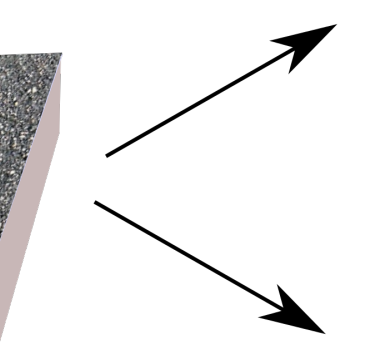

Increased resistivity

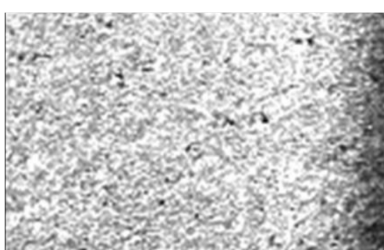

400

300

200

ᄀ

100

0 